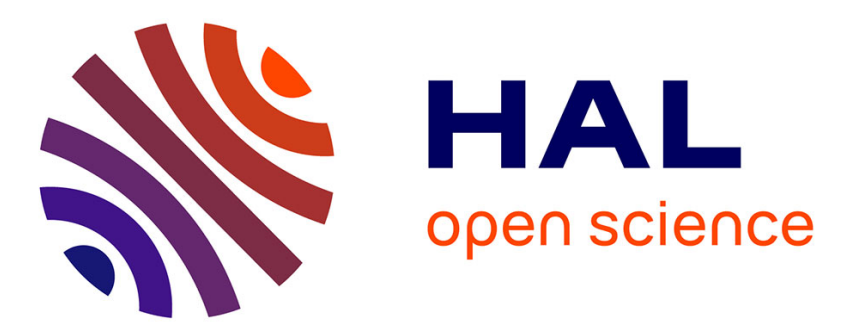

\title{
Essai de validation d'une méthode d'échantillonnage linéaire appliquée à trois espèces de rongeurs d'un peuplement de micromammifères d'un milieu saharien (Guelmime, Maroc)
}

\author{
A.K. Zaime, Abdel Kadder Zaime, Michel Pascal
}

\section{To cite this version:}

A.K. Zaime, Abdel Kadder Zaime, Michel Pascal. Essai de validation d'une méthode d'échantillonnage linéaire appliquée à trois espèces de rongeurs d'un peuplement de micromammifères d'un milieu saharien (Guelmime, Maroc). Mammalia, 1988, 52 (2), pp.243-258. 10.1515/mamm.1988.52.2.243. hal-02196144

\section{HAL Id: hal-02196144 \\ https://hal.science/hal-02196144}

Submitted on 19 Sep 2019

HAL is a multi-disciplinary open access archive for the deposit and dissemination of scientific research documents, whether they are published or not. The documents may come from teaching and research institutions in France or abroad, or from public or private research centers.
L'archive ouverte pluridisciplinaire HAL, est destinée au dépôt et à la diffusion de documents scientifiques de niveau recherche, publiés ou non, émanant des établissements d'enseignement et de recherche français ou étrangers, des laboratoires publics ou privés. 


\title{
Essai de validation d'une méthode d'échantillonnage linéaire appliquée à trois espèces de rongeurs d'un peuplement de micromammifères d'un milieu saharien (Guelmime, Maroc)
}

\author{
par Abdel Kadder ZAIME ${ }^{1}$ et Michel PASCAL ${ }^{2}$
}

\author{
'Laboratoire des Vertébrés de l'Institut Agronomique et Vétérinaire Hassan II, \\ Complexe d'Agadir, B.P. 121, Aït Melloul, Maroc \\ ${ }^{2}$ Laboratoire de la Faune Sauvage de l'Institut National Agronomique, \\ 78350 Jouy-en-Josas, France
}

Summary. - In the view to work out an integrated pest management system against 3 rodent species (Meriones shawi, Gerbillus nanus, Psammomys obesus), pest of the saharian agriculture of Morocco, we had to perfect a sampling method of these mammals both light and relevent to the question. Two separate experimentations were undertaken to test a sampling method founded on trapping out on a strip. The first step consists of the analysis of data provided by Capture-Marking-Recapture operation of all the rodents on a 1 ha $(100 \times 100 \mathrm{~m})$ quadrat, subdivided in $10 \times 10 \mathrm{~m}$ unities. This operation allowed us to determine rationally the geometric characteristics of the strip, that is to say, a $100 \times 10 \mathrm{~m}$ strip made of 10 adjoining squares of $10 \mathrm{~m}$ side, each equiped with 4 Manufrance traps. During the second step of this experimentation, two sampling strips were superposed on the quadrat system and all the trapped rodents were removed. This operation allowed us to fixe the general timing of operations and, particularly, those of traps checking. Samplings by trapping out must be performed within 4 days with 2 daily trap checkings. These last characteristics were precised by results of a second experimentation wich consist of setting up 16 sampling strips. The limits of this linear trapping out sampling method are discussed mainly in the light of behavioural and landscape factors.

Résumé. - Pour développer une stratégie de lutte intégrée à l'encontre de 3 espèces de rongeurs (Meriones shawi, Gerbillus nanus, Psammomys obesus), ravageurs de cultures de la zone saharienne marocaine, il importait de mettre au point une méthode d'échantillonnage de ces mammifères qui soit à la fois légère et pertinente à l'égard de la question posée. Deux expériences distinctes ont été menées. La première a consisté à réaliser, au cours d'une première étape, sur un quadrat de un hectare de maille $10 \mathrm{~m} \times 10 \mathrm{~m}$ la capture, le marquage et la recapture de tous les rongeurs. Elle a permis de fixer rationnellement les caractéristiques géométriques de la bande de piégeage $(100 \mathrm{~m} \times 10 \mathrm{~m}$ subdivisée en carrés unitaires de $10 \mathrm{~m} \times 10 \mathrm{~m}$ équipés chacun de 4 ratières Manufrance). Au gré d'une deuxième étape, grâce au piégeage exhaustif de deux bandes échantillon superposées à ce quadrat, il a été possible d'opter de façon fondée pour une séquence temporelle des opérations d'échantillonnage. Ces résultats ont été enrichis par l'analyse de ceux provenant d'une deuxième expérience indépendante consistant en la pose de 16 bandes de pié-

Mammalia, t. 52, $n^{\circ} 2,1988$. 
geage. Ils aboutissent à proposer un contrôle biquotidien des bandes pendant 4 jours consécutifs. La discussion porte sur les limites que l'on peut attendre d'une pareille méthode et sur l'influence probable de divers éléments du paysage ou d'origine comportementale sur les résultats fournis par cette méthode.

\section{I) INTRODUCTION}

Outre leur rôle de réservoir dans le cycle de plusieurs parasitoses à incidence humaine, entre autres Leishmania major (Rioux et al. 1982, 1984) et Schistosoma mansoni, plusieurs espèces de rongeurs champêtres constituent au Maroc, et plus généralement pour l'ensemble des pays du Maghreb, des déprédateurs notables de cultures et de pâturages. En dépit du fait que les dégâts imputables à ces rongeurs n'aient pas, à ce jour, fait l'objet d'évaluations précises, il est établi que plusieurs espèces, souvent sympatriques, sont concernées à des degrés divers et réalisent des attaques généralement non synchrones sur l'ensemble du territoire.

Dans le but de réaliser une prévision des explosions démographiques de ces mammifères afin de développer une stratégie fondée de régulation de leurs effectifs, il importe en premier lieu de pouvoir décrire et quantifier, dans toute la mesure du possible, la cinétique de leurs populations. Cette simple approche descriptive nous permettrait, entre autres, de déterminer si ces populations réalisent des cycles de pullulation au sens de Krebs (Krebs et Myers 1974; Taitt et Krebs 1985), ou sont plus simplement sous la dépendance étroite, directe ou indirecte, de facteurs limitants trophiques ou climatiques tels que l'eau (Zaime 1985).

Réaliser une description quantifiée des cycles d'abondance suppose que nous dịsposions de méthodes d'échantillonnage standardisées, fiables, légères, adaptées à la biologie des espèces et utilisables sur la majorité des agroécosystèmes qui les abritent.

Les quelques travaux consacrés au suivi diachronique d'effectifs de rongeurs en milieu sub-désertique africain ont essentiellement utilisé la méthode des quadrats avec " capture-marquage-recapture " (CMR) (Daly et Daly 1973, 1974, 1975 ; Poulet 1982 ; Zaime 1985). Cette méthode offre l'avantage indéniable d'apporter des informations fondamentales au niveau de l'individu. Cependant, à effort de piégeage égal, elle se révèle, à plusieurs égards, moins performante au plan des informations recherchées par le dynamicien des populations que le piégeage exhaustif linéaire (Spitz 1974 ; Rodolphe et Pascal 1985 ; Pascal et Meylan 1986). En outre, elle exclut très souvent le sacrifice des sujets capturés et interdit donc l'accès à l'évaluation de paramètres démographiques. Ces diverses raisons nous ont incités à opter pour le développement d'une méthode d'échantillonnage linéaire exhaustive.

C'est donc dans le but d'acquérir une méthode d'échantillonnage linéaire standardisée pour 3 espèces de Gerbillidés sahariens, Meriones shawi (Duvernoy, 1842), Gerbillus nanus Blanford, 1875 et Psammomys obesus Cretzschmar, 1828, que nous avons réalisé deux expérimentations sur deux milieux distincts de la région de Guelmime, le long de l'oued Sayad (Fig. 1).

Une première expérience, destinée à évaluer la pertinence du choix des caractéristiques géométriques du système de piégeage, a associé la technique des quadrats à celle des bandes échantillons. Elle a permis la capture simultanée de $58 \mathrm{M}$. shawi, 82 G. nanus, 3 G. campestris Levaillant, 1857, 57 P. obesus, 1 Eliomys occidentalis (d'après Petter 1961) et 3 Elephantulus rozeti (Duvernoy, 1833). 
Une deuxième étape expérimentale, destinée à préciser les caractéristiques temporelles du piégeage, s'est déroulée en 5 séquences mensuelles de piégeage, du mois de février au mois de juin 1986, entraînant la pose de 16 bandes échantillons qui ont permis la capture de $103 \mathrm{M}$. shawi, $128 \mathrm{G}$. nanus et $91 \mathrm{P}$. obesus.

\section{II) MATÉRIEL ET MÉTHODE}

\section{1) DESCRIPTION SUCCINCTE DU SITE D'ÉTUDE}

La plaine de Guelmime, située au sud-ouest de l'Anti-Atlas, est la vallée de l'oued Sayad qui inonde 3 à 4 fois par an, entre les mois de novembre et de mars, de vastes superficies.

Les sites d'étude (Fig. 1) sont situés dans le fond de cette plaine alluviale, à une altitude avoisinant $250 \mathrm{~m}$. Le premier est implanté sur un parcours pastoral à dromadaires, moutons et chèvres. Son couvert végétal comprend 3 strates.
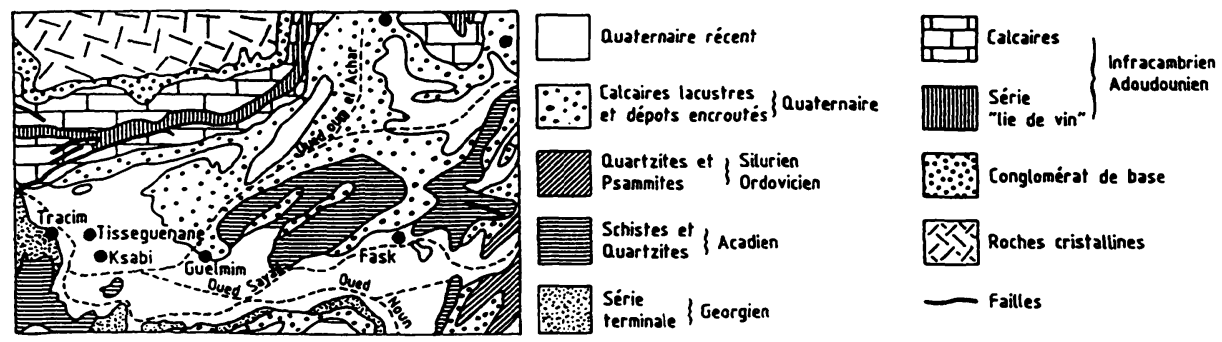

Esquisse géologique du bassin Seyad-Noun (d'après

la carte au 1/500000 du service géologique du Maroc).

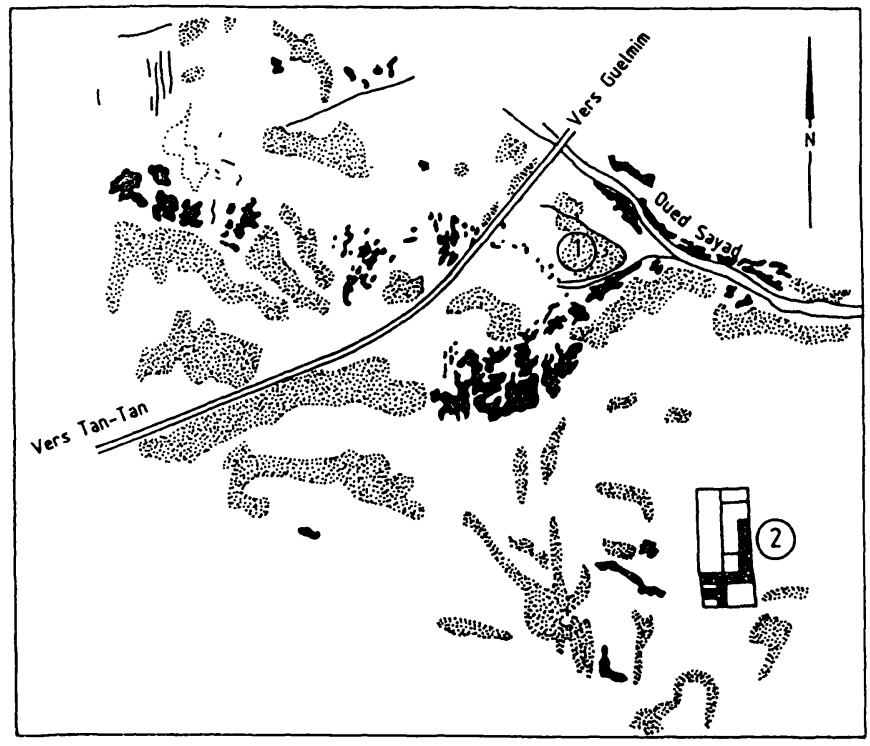

Tamarix

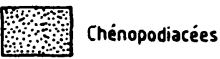

T) oued

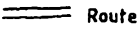

(1) Milieu pastoral

(2) Milieu cultivé

Fig. 1. - Implantation du site d'étude de Guelmime. 
La première, herbacée, a une existence éphémère conditionnée par le régime imprévisible des crues et des pluies. La seconde, buissonneuse, pérenne, fixée sur des dunes fluviatiles, est constituée essentiellement de Chénopodiacées (Salsola vermiculata, Atriplex halimus et Suaeda fruticosa). Enfin, localisée le long de l'oued, une strate arbustive de Tamarix gallica est qualifiée par Dijon (1963) de "forêt-galerie ". Le second site de travail est implanté sur une exploitation agricole qui s'adonne essentiellement à la céréaliculture et entretient de petites surfaces de luzerne. Ce milieu agraire, défriché depuis peu sur un parcours pastoral, a laissé de place en place des lambeaux de l'association végétale originale et abrite sur les surfaces cultivées plusieurs espèces adventices.

Les relevés (1979-1986) de la station météorologique de Tagant, localisée à $35 \mathrm{~km}$ de Guelmime, ont permis d'établir la valeur du coefficient d'Emberger

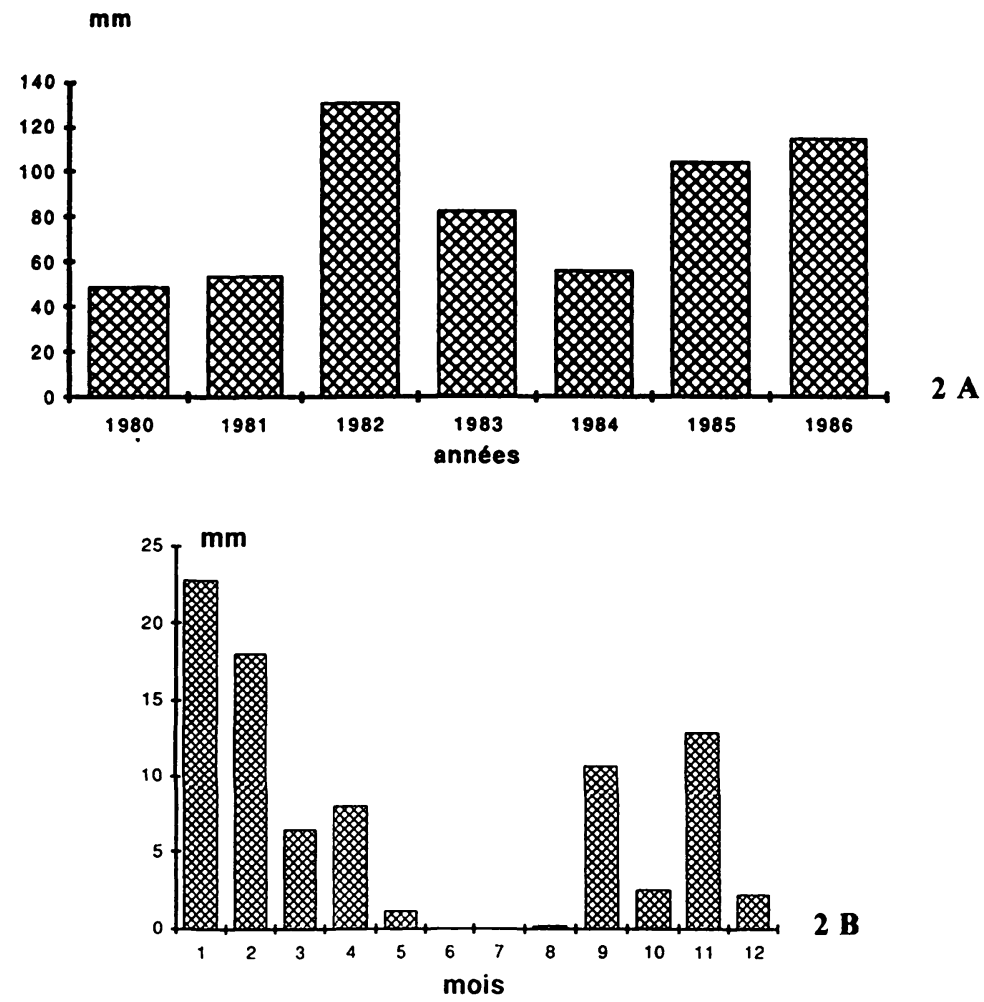

Fig. 2. - Précipitations enregistrées à Tagant de 1980 à 1986 annuellement (Fig. 2a) et mensuellement (Fig. 2b).

$(Q=10)$ qui situe le site dans l'étage climatique saharien caractérisé à la fois par de faibles précipitations, d'importance variable d'une année sur l'autre, survenant irrégulièrement pendant les mois d'hiver (Fig. 2), et par de fortes températures estivales associées à de grands écarts thermiques circadiens. 


\section{2) CONDUITE DES EXPÉRIMENTATIONS}

Du 10 au 26 juillet 1986, une première expérimentation a été menée en 3 étapes sur le milieu pastoral. Au cours d'une première étape un quadrat d'un ha $(100 \mathrm{~m} \times 100 \mathrm{~m})$, subdivisé en mailles carrées de $10 \mathrm{~m} \times 10 \mathrm{~m}$, a été installé (Fig. 3). Le choix de la taille de la maille a été guidé par la valeur des "distances entre recaptures successives " (DRS) obtenues à l'occasion d'un premier travail (Zaime 1985) sur M. shawi, G. campestris et Lemniscomys barbarus. Sur chacun des carrés élémentaires, 2 ratières Manufrance appâtées à la datte ont été placées au niveau des passages de rongeurs et au voisinage des entrées de terriers, le plus souvent sous la protection des buissons de $S$. vermiculata afin d'éviter une exposition prolongée des pièges au soleil, souvent fatale aux captures. En dépit de cette précaution, nous avons cependant enregistré quelques pertes (Tab. 1). Les pièges ont été contrôlés biquotidiennement, le matin et le soir, pendant 6 jours et tous les micromammifères capturés ont été pesés, sexés, marqués individuellement par amputation de phalanges puis relâchés à leur emplacement de capture.

A l'issue de cette première phase, tous les pièges du quadrat furent fermés à l'exception de ceux de 2 bandes parallèles distantes de $40 \mathrm{~m}$ (bandes $\mathrm{X}_{3}$ et $\mathrm{X}_{8}$ de la Fig. 3A). Le dispositif de piégeage de ces bandes a été renforcé par l'adjonction de 2 pièges supplémentaires par carré élémentaire et a été contrôlé biquotidiennement pendant 5 jours consécutifs. Tous les sujets capturés ont été sacrifiés, autopsiés afin de déterminer leur état sexuel, et leurs globes oculaires ont été prélevés pour établir ultérieurement les structures d'âge des diverses populations.

Enfin, pendant les 5 derniers jours de l'expérimentation, tous les pièges du quadrat, y compris ceux des bandes $X_{3}$ et $X_{8}$, furent réarmés, contrôlés biquotidiennement et l'ensemble des captures fut sacrifié aux fins d'autopsies.

Une deuxième expérimentation a consisté à établir mensuellement, de février à juin 1986,3 à 4 bandes de piégeage standard de $100 \mathrm{~m} \times 10 \mathrm{~m}$ subdivisées en carrés élémentaires de $10 \mathrm{~m} \times 10 \mathrm{~m}$ équipés chacun de 4 ratières Manufrance appâtées à la datte et contrôlées biquotidiennement pendant 10 jours consécutifs. Tous les sujets capturés ont été sacrifiés et autopsiés. Chaque mois, une ou deux bandes-échantillons ont été posées simultanément sur le milieu pastoral et le milieu agraire.

\section{III) RÉSULTATS. DISCUSSION}

\section{A) L'expérimentation sur quadrat}

\section{1) LA PHASE "CAPTURE-MARQUAGE-RECAPTURE "}

L'opération de CMR a été menée au moyen de 200 pièges contrôlés 12 fois, offrant ainsi un total de 2400 possibilités de capture. 123 micromammifères différents ont été capturés au moins une fois (Tab. 1), nombre représentant $62 \%$ de la capacité de piégeage instantané du quadrat. En outre, le nombre total de captures et de recaptures réalisées au cours de cette première phase expérimentale s'élève à 246 (Tab. 1 ; Fig. 3A), représentant un taux de remplissage de $10,3 \%$ de la capacité totale de piégeage. Ces deux résultats laissent, supposer 


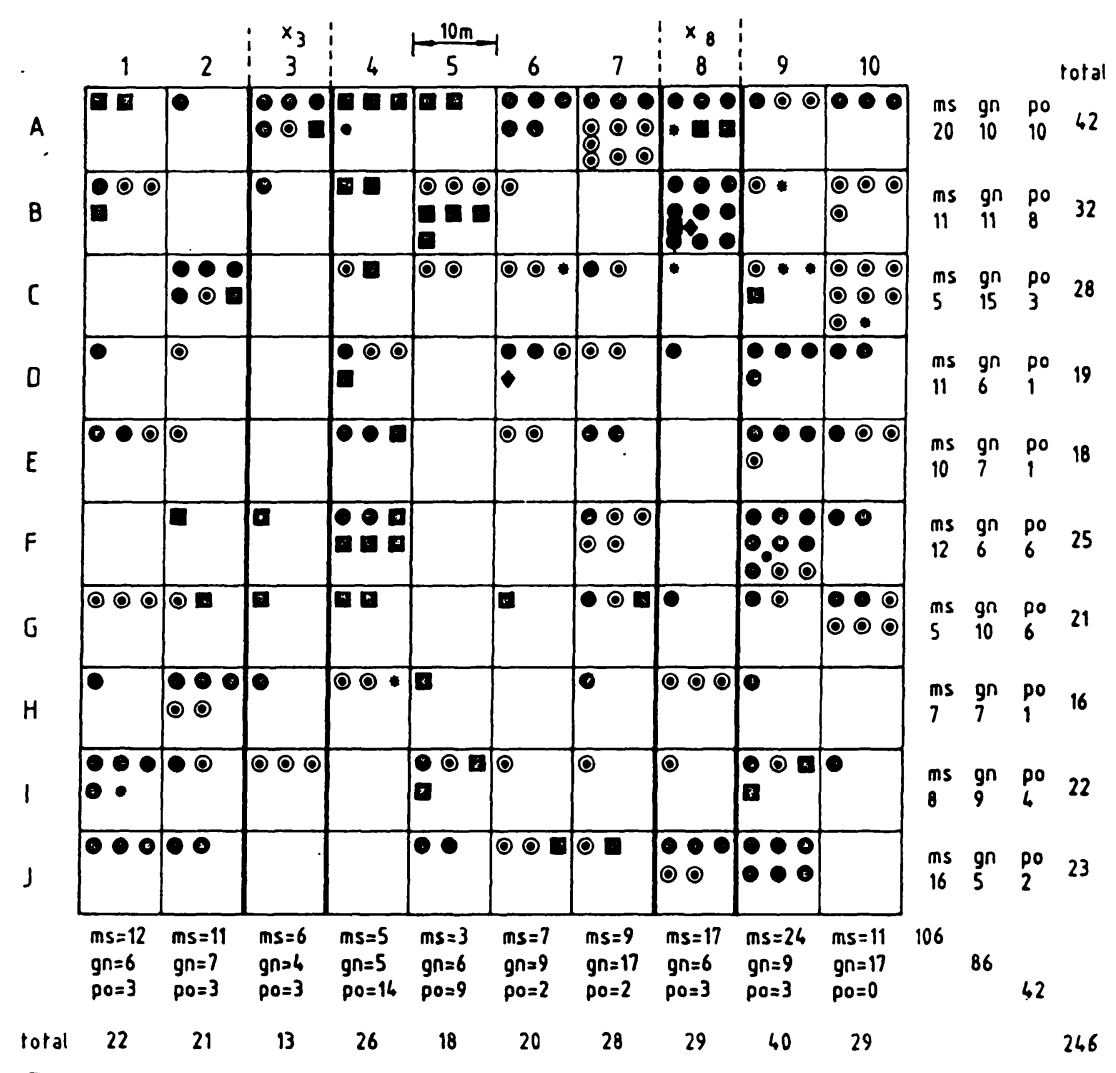

(A)

(B)

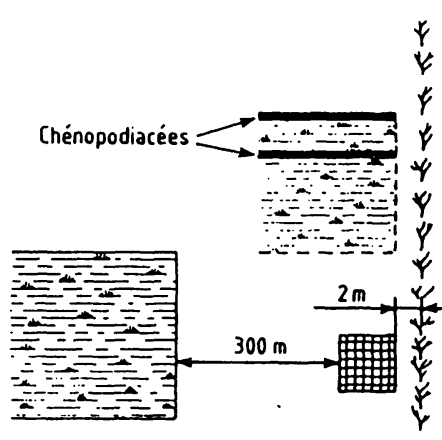

- Meriones shawi (ms )

OGerbillus nanus (gn)

- Gerbillus campestris

Exammomys obesus ( po )

- Elephantulus rozeti

- Eliomys occidentalis

* Tamarix gallica

三éréale

囲Quadrat

Fig. 3. - Localisation des captures sur le quadrat (A) et implantation du quadrat par rapport à certains éléments du paysage (B). 
TABLEAU 1. - Récapitulatif de l'ensemble des captures et recaptures réalisées sur le quadrat. Le nombre total de captures et recaptures $\left(\mathrm{N}_{t}\right)$ est égal à la somme du nombre de sujets capturés marqués $\left(\mathrm{N}_{\mathrm{c}}\right)$ et du nombre de sujets morts dès leur première capture $\left(\mathrm{N}_{\mathrm{nm}}\right)$ au nombre total de recaptures $\left(\mathrm{N}_{\mathrm{r}}\right) . \mathrm{N}_{\mathrm{nm}}$ : nombre de morts marqués.

Especes

\begin{tabular}{lccccc} 
Meriones shawi & 30 & 6 & 6 & 69 & 105 \\
Psommomys obesus & 28 & 5 & 1 & 9 & 42 \\
Gerbillus nanus & 43 & 4 & 1 & 39 & 86 \\
Gerbillus compestris & 3 & 0 & 0 & 5 & 8 \\
$\begin{array}{l}\text { Eliomys occidentalis } \\
\text { Elephantulus rozeti }\end{array}$ & 1 & 0 & 0 & 1 & 2 \\
\multicolumn{1}{c}{ Total } & 108 & 15 & 8 & 123 & 246
\end{tabular}

avec quelque raison que les capacités de piégeage du dispositif expérimental ont été amplement suffisantes pour assurer la capture de la totalité des micromammifères capturables, inféodés à la surface drainée par le quadrat.

La figure $3 \mathrm{~B}$ situe le quadrat par rapport à certains éléments importants du paysage. La figure $3 \mathrm{~A}$ localise, par carré élémentaire, la totalité des captures et recaptures enregistrées sur le quadrat, espèce par espèce. L'examen superficiel de cette dernière figure ne permet pas d'affirmer $a$ priori qu'il existe un partage net de l'espace par ces diverses espèces.

Pour déterminer si, sur l'ensemble de la superficie du quadrat, certaines zones sont plus fréquentées que d'autres, nous avons comparé, grâce à un test de $\chi^{2}(9 \mathrm{ddl})$, le nombre moyen de captures et recaptures par colonne ou ligne, respectivement caractérisées par les indices numériques ou alphabétiques de la fig. $3 \mathrm{~A}$, à leur nombre réel. Le calcul a d'abord porté sur la totalité des observations, puis sur chacune des 3 espèces les plus abondantes (Tab. 2). Pour l'une d'entre elles $(P$. obesus), la valeur moyenne des captures et recaptures par ligne et colonne est inférieure à 5 , effectif qu'il est nécessaire d'atteindre pour que

TABLEAU 2. - Evaluation de l'homogénéité de la répartition spatiale des captures par comparaison au moyen d'un test de $\chi^{2}$ des valeurs observées, à la moyenne des captures $(\mathrm{N})$ sur les lignes ou les colonnes du quadrat.

$\begin{array}{lccc} & N & H^{2} \text { (lignes) } & H^{2} \text { (colonnes) } \\ \text { rotal des captures } & 24,7 & 21,0 & 21,8 \\ \text { Meriones showi } & 10,6 & 17,6 & 33,1 \\ \text { Gerbillus nanus } & 8,6 & 9,6 & 23,1 \\ \text { Psammomys obesus } & 4,2 & 21,8 & 36,6\end{array}$

le test ait une certaine robustesse. Dans une perspective de simple comparaison et en raison de la grande cohérence de l'ensemble de ces résultats, nous avons conservé les valeurs associées à cette espèce. En effet, les $\chi^{2}$ observés sont tous élevés et, à l'exception de 2 , ils sont supérieurs à 19 , niveau qui a une probabilité de 0,025 d'être dépassée. La surface du quadrat n'est donc pas uniformément fréquentée. En particulier la frange contiguë à la zone de Tamarix gallica repré- 
sente selon toute vraisemblance un lieu d'élection pour les rongeurs, de même qu'il semble exister un certain " tropisme " positif pour les parcelles céréalières. Pour évaluer la pertinence de ces hypothèses, nous avons réalisé un test de sphéricité (Mauchly 1940) sur les déplacements. Nous avons limité son application à $M$. shawi et $G$. nanus, seules espèces dont le nombre de recaptures permettait l'analyse. Le test ne permet pas de rejeter l'hypothèse d'isotropie des déplacements pour $M$. shawi $\left(\chi^{2}\right.$ (obs.) $\left.=1,38 ; \mathrm{ddl}=2\right)$. En revanche, les déplacements de $G$. nanus sont significativement $(0,5 \%)$ orientés préférentiellement selon une direction parallèle à la lisière de Tamarix gallica $\left(\chi^{2}\right.$ (obs.) $=5,61 ; \mathrm{ddl}=2$ ).

Une façon d'évaluer l'adéquation d'une technique de piégeage à une espèce consiste à analyser le tableau des captures et recaptures de la phase de CMR (Tab. 3). Dans un premier temps nous avons cherché à déterminer si l'efficacité du piégeage était homogène dans le temps, ou s'il nous fallait supposer une période minimale d'adaptation à la présence des pièges ou au dérangement occasionné par leur mise en place. Pour cela nous avons comparé, à l'aide d'un

TABLEAU 3. - Calendrier quotidien des captures et recaptures réalisées sur le quadrat. Certains individus ayant été contrôlés deux fois au cours de la même journée, le nombre de recaptures est généralement inférieur à celui présenté dans le tableau $1(C$ : capturés ; $R$ : recapturés ; $\mathrm{T}=\mathrm{C}+\mathbf{R})$.

\begin{tabular}{|c|c|c|c|c|c|c|c|c|c|c|c|c|c|c|c|c|c|c|}
\hline \multirow[b]{2}{*}{ Date } & \multicolumn{3}{|c|}{ M. shawi } & \multicolumn{3}{|c|}{ G. nonus } & \multicolumn{3}{|c|}{ P.obesus } & \multicolumn{3}{|c|}{ G. campes. } & \multicolumn{3}{|c|}{ E.occiden. } & \multicolumn{3}{|c|}{ E. rozeti } \\
\hline & c & $\mathbf{R}$ & $\mathbf{T}$ & C & R & $\mathbf{T}$ & C & R & $\mathbf{T}$ & C & R & $\mathbf{I}$ & C & R & $\mathbf{T}$ & C & R & $\mathbf{T}$ \\
\hline 10 & 10 & - & 10 & 8 & - & 8 & 3 & - & 3 & 0 & - & 0 & 0 & - & o & 1 & - & o \\
\hline 11 & 5 & 4 & 9 & 8 & 2 & 10 & 7 & o & $?$ & 1 & o & 1 & 0 & o & 0 & 0 & 0 & 0 \\
\hline 12 & 4 & 10 & 14 & 4 & 6 & 10 & 4 & 1 & 5 & 0 & 0 & 0 & 0 & 0 & $\mathbf{0}$ & 0 & 0 & o \\
\hline 13 & 6 & 6 & 12 & 13 & 8 & 21 & 5 & 1 & 6 & 2 & 1 & 3 & 1 & 0 & 1 & 0 & 0 & 0 \\
\hline 14 & 3 & 9 & 12 & 4 & 13 & 17 & 6 & 1 & 7 & 0 & 1 & 1 & 0 & 1 & 1 & 2 & 0 & 2 \\
\hline 15 & 2 & 12 & 14 & 6 & 9 & 15 & 3 & 4 & 7 & 0 & 2 & 2 & o & o & o & 0 & 0 & 0 \\
\hline Totol & 30 & 41 & 71 & 43 & 38 & 81 & 28 & 7 & 35 & 3 & 4 & 7 & 1 & 1 & 2 & 3 & 0 & 3 \\
\hline
\end{tabular}

test de $\chi^{2}$, le nombre moyen quotidien de captures et recaptures, aux valeurs observées. Les $\chi^{2}$ calculés s'élèvent respectivement à $1,76 / 2,20 / 9,21$ pour $M$. shawi, $P$. obesus et $G$. nanus. Pour les deux premières espèces, le nombre de captures et recaptures quotidiennes n'est pas significativement différent de la moyenne. En revanche, cette affirmation n'est pas valable pour $G$. nanus $\left(P\left(\chi^{2}\right)<9,2=0,1 ; \mathrm{ddl}=5\right)$, espèce dont la fréquence de capture augmente singulièrement à partir du quatrième jour de piégeage. Ce fait peut être interprété comme une défiance passagère du rongeur à l'égard des pièges, comportement moins marqué chez $M$. shawi et $P$. obesus.

Ce comportement peut à nouveau être évoqué pour expliquer l'évolution irrégulière du nombre de captures nouvelles au cours du temps (Tab. 3). Ce phénomène, particulièrement net chez $G$. nanus, s'atténue chez $P$. obesus et demeure discret chez $M$. shawi. En outre, des sujets nouveaux apparaissent encore le dernier jour de piégeage, ce qui laisse supposer que le temps de piégeage adopté dans notre protocole ne permet pas la capture de la totalité des individus 
piégeables. Enfin, la fréquence de recapture des individus recapturables (Fig. 4) est élevée pour $M$. shawi, moins bonne pour $G$. nanus et mauvaise pour $P$. obesus. En conséquence, seul $M$. shawi serait justiciable d'une étude de cinétique de population par la technique des CMR employant le système de piégeage décrit ci-dessus.

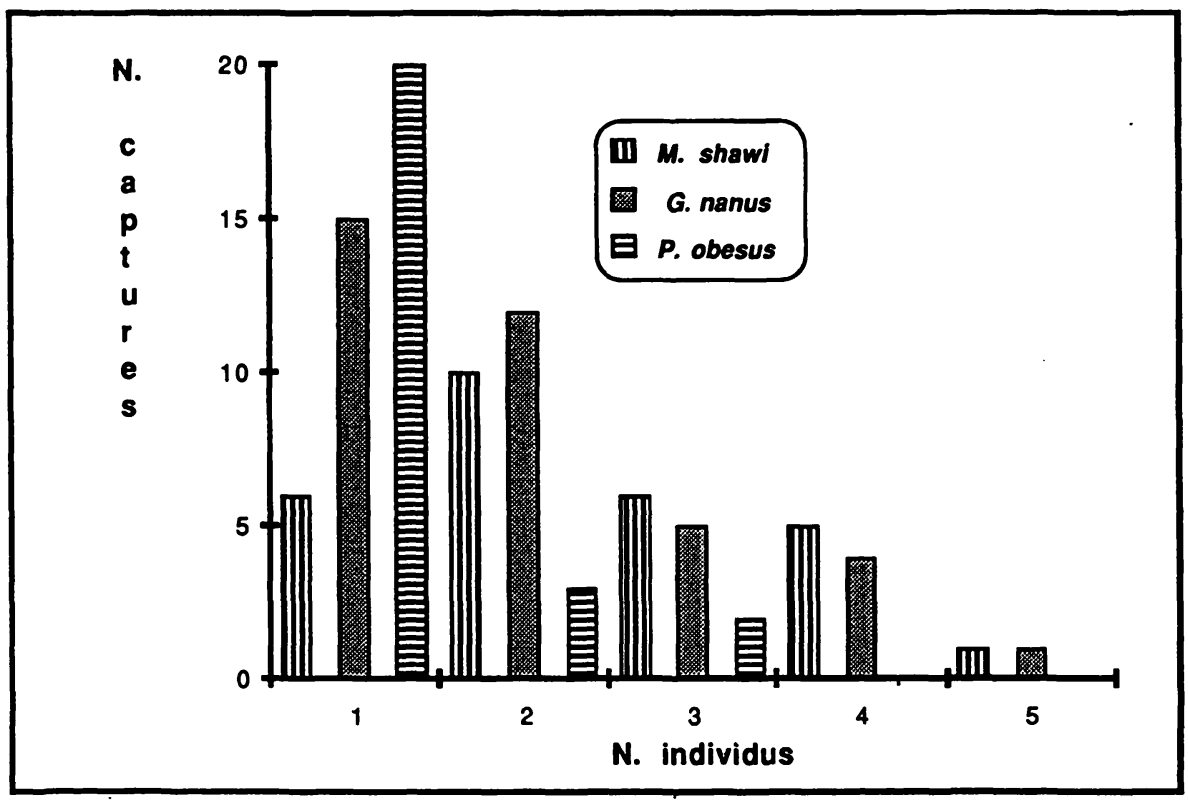

Fig. 4. - Fréquence des individus ayant été capturés et recapturés $\mathrm{N}$ fois.

Afin de détecter une éventuelle efficacité différentielle du piégeage en fonction du sexe, nous avons comparé, pour chaque espèce, la représentation de chaque sexe dans l'effectif des captures à la répartition théorique égalitaire. La valeur des tests de $\chi^{2}$ à 1 ddl associés aux résultats de la phase de CMR conduit à rejeter l'hypothèse d'une efficacité différentielle du piégeage pour $M$. shawi (12 o", $18 \% ; \chi^{2}$ (obs.) $\left.=1,20\right)$ et $P$. obesus $\left(12 \circ, 16 \% ; \chi^{2}\right.$ (obs.) $\left.=0,57\right)$ et à l'accepter, au seuil de 0,5\%, pour $G$. nanus $\left(33 \circ, 10 \% ; \chi^{2}\right.$ (obs.) $=12,30$ ), à moins de supposer, chez cette dernière espèce, une représentation des sexes non équilibrée dans la population échantillonnée, hypothèse à notre connaissance jamais vérifiée à ce niveau de probabilité chez les mammifères. Nous avons étendu cette analyse à l'ensemble des captures réalisées au gré des $\mathbf{3}$ phases de l'expérimentation et abouti à des conclusions tout à fait similaires (M. shawi : $27 \mathrm{O}^{\circ}, 31$ ९; $\chi^{2}$ (obs.) $=0,28$. P. obesus : $30 \sigma^{\circ}, 27 \% ; \chi^{2}$ (obs.) $=0,16$. G. campestris : $57 o^{\circ}, 25 \% ; \chi^{2}$ (obs.) $=12,49$ ). La poursuite de cette analyse voudrait que nous cherchions à savoir si l'efficacité du piégeage se fait sentir différemment selon les diverses classes d'âge. Nous ne disposons pas à ce jour des courbes étalons, poids du cristallin sec/âge, relatives aux espèces qui nous intéressent ici. Aussi ne pouvons-nous aborder cet aspect du problème de façon pertinente.

Pour évaluer l'impact du piégeage sur les sujets capturés, nous avons comparé le poids initial, relevé au marquage lors de la première capture, au poids final, 
enregistré à l'occasion des autopsies réalisées au cours des 3 phases expérimentales. Quel que soit l'individu, le sexe ou l'espèce, le poids total chute entre la première et la dernière capture. Sur $30 \mathrm{M}$. shawi marqués, 20 furent autopsiés et témoignèrent d'une chute pondérale moyenne de $20,1 \mathrm{~g}$ représentant $20,4 \%$ de la biomasse initiale. Au nombre des $43 \mathrm{G}$. nanus marqués, 22 furent autopsiés, enregistrant une chute pondérale moyenne de $8,8 \mathrm{~g}$ représentant $32,0 \%$ de la biomasse initiale. Enfin, les $8 P$. obesus autopsiés parmi les 28 marqués, ont perdu en moyenne $23,9 \mathrm{~g}$ ce qui représente $13,1 \%$ de la biomasse initiale. Afin d'éliminer les éventuelles pertes de poids, indépendantes du piégeage et liées aux activités de lactation, gestation et mise-bas, nous avons repris ces calculs sur la seule fraction mâle de l'échantillon, en se limitant à la seule espèce $M$. shawi pour des raisons d'effectifs. Nous obtenons des résultats tout à fait comparables aux précédents, à savoir une perte de poids moyenne, sur 9 sujets, de $17,1 \mathrm{~g}$, représentant $21,1 \%$ de la biomasse initiale. En dépit donc de toutes les précautions prises pour limiter le temps de séjour des animaux dans les pièges par la réalisation d'un contrôle quasi permanent en matinée $(7 \mathrm{~h}-10 \mathrm{~h})$ et en fin d'après-midi ( $16 \mathrm{~h}-19 \mathrm{~h})$, nous n'avons pu éviter de perturber fortement les sujets capturés.

L'analyse des valeurs de « distances moyennes entre capture et recaptures successives " (DRS) permet d'évaluer, en première approximation, l'adéquation de la dimension des mailles d'un quadrat au comportement général du déplacement d'une espèce (Spitz 1974 ; Duplantier et al. 1984). Il convient cependant, d'une part d'éviter la confusion entre DRS et domaine vital, d'autre part de percevoir qu'il y a une relation étroite entre la valeur de la DRS et la dimension de la maille d'un quadrat (Duplantier et al. 1984). En effet, si le piège est correctement adapté à l'espèce, il se comporte comme un " interrupteur ", pouvant intercepter l'animal avant qu'il ait atteint les limites de son domaine vital. Si la distance entre les pièges est faible au regard des dimensions du domaine vital, son évaluation par la DRS sera donc plus ou moins sous-estimée et différera peu de celle de la maille du quadrat. Dans la situation inverse, seuls certains animaux auront la possibilité d'être capturés et ceci en un seul point : la valeur de la DRS tendra alors rapidement vers zéro. Enfin, il importe de trouver une adéquation entre la densité de pièges, la densité maximale de rongeurs et la fréquence des contrôles. En effet, un piège occupé par un sujet ne peut en accueillir un second. Au gré des captures, la taille réelle de la maille du système de piégeage augmente entre 2 contrôles. Dans la perspective d'optimiser le nombre de captures afin de disposer d'une méthode d'échantillonnage performante, il importe donc de choisir une maille de dimension inférieure à celle du domaine vital de l'espèce afin d'offrir une bonne probabilité de capture à chaque individu inféodé au quadrat. Par ailleurs, il importe de tester cette maille en période de forte densité, situation de la présente expérimentation, afin de diagnostiquer une éventuelle

TABLEAU 4. - Le calcul de la distance moyenne entre captures et recaptures successives (DRS) et son écart type (E.T.) a été établi pour chaque espèce à partir d'un ensemble de mesures de la distance entre captures et recaptures $\left(\mathrm{N}_{\text {DRS }}\right)$ associées aux individus capturés au moins une fois $\left(\mathbf{N}_{\mathrm{i}}\right)$.

$\begin{array}{lcccc} & N_{1} & N_{\text {ORS }} & \text { OMR } & \text { E.T. } \\ \text { M. showi } & 22 & 69 & 12,15 & 12,90 \\ \text { G. nonus } & 21 & 39 & 19,11 & 13,47 \\ \text { P. obesus } & 5 & 9 & 19,95 & 15,00 \\ \text { G. compestris } & 1 & 5 & 10,90 & 4,30\end{array}$


saturation du système de piégeage, éventualité qui, nous l'avons vu précédemment, ne se présente pas ici. Il nous faut donc vérifier que la DRS associée à chacune des espèces capturées sur le quadrat est strictement supérieure à la dimension de la maille élémentaire, elle-même inférieure à $10 \mathrm{~m}$ puisque nous disposons de 2 pièges par carré élémentaire. Cette condition est vérifiée pour les 3 espèces dominantes (Tab. 4).

\section{2) COMPARAISON DE LA PHASE CMR \\ Ȧ LA PHASE “CAPTURES EXHAUSTIVES SUR BANDES » (CEB)}

Cette deuxième phase expérimentale est essentiellement destinée d'une part à tester l'hypothèse d'une meilleure efficacité du piégeage linéaire par rapport au piégeage sur surface compacte, d'autre part à évaluer cette éventuelle amélioration d'efficacité.

Sur chaque bande-échantillon comportant 40 pièges contrôlés 10 fois, 45 rongeurs ont été capturés, toutes espèces confondues. Le taux d'occupation des pièges s'élève donc à $11,2 \%$, valeur légèrement supérieure à celle obtenue sur le quadrat $(10,3 \%)$, mais insuffisante pour parler de saturation du dispositif de piégeage.

Les rendements des 2 dispositifs de piégeage ont été calculés sur la base des seuls effectifs, à l'exclusion des recaptures pour la phase de CMR. Ils ont été évalués par le rapport du nombre de captures au nombre de pièges, ou par celui du nombre de captures au nombre de pièges contrôlés (Tab. 5). Les

TABLEAU 5. - Effectifs et taux d'efficacité des systèmes de piégeage sur quadrat et sur bande.

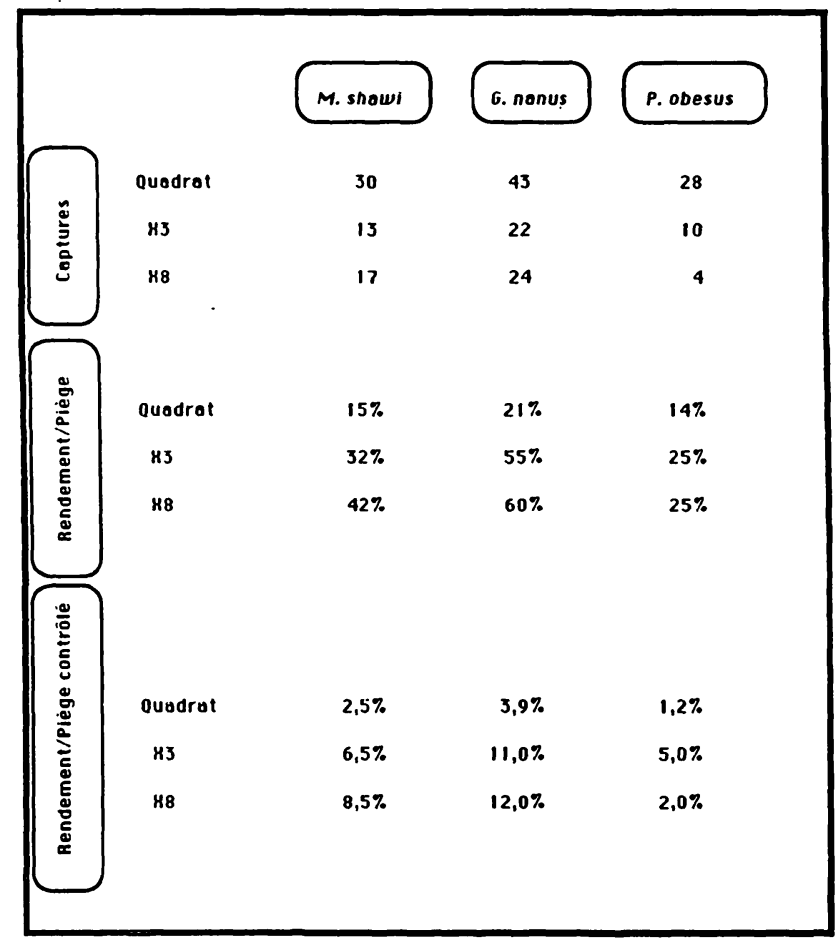


résultats de ces calculs témoignent tous d'une augmentation sensible de l'efficacité du piégeage sur bande qui peut, dans certains cas, plus que doubler.

Sur l'ensemble des 2 bandes-échantillon, 15 des $30 \mathrm{M}$. shawi, 20 des 46 $G$. nanus et 5 des $14 P$. obesus capturés ne sont pas marqués. Ce résultat peut recevoir deux interprétations simples. Soit certains sujets sont préférentiellement capturés et immobilisent des pièges situés dans des zones privilégiées de capture, interdisant ainsi la capture d'autres rongeurs présents, soit, plus simplement, l'expérience se situant en période de forte densité, les domaines libérés par les animaux capturés sont très rapidement réoccupés. Le test de cette dernière hypothèse peut être réalisé par l'analyse de l'évolution, au cours du temps, du nombre de captures. Nous ne l'avons pas réalisé sur les résultats de la présente expérience en raison de la perturbation apportée à la population lors de la phase de CMR, mais nous l'avons appliqué aux résultats de la deuxième expérimentation menée sur bandes-échantillon.

\section{B) Analyse des piégeages mensuels sur bandes}

Sur la figure 5 sont représentés les effectifs de $M$. shawi, $P$. obesus et $G$. nanus capturés quotidiennement sur 16 bandes-échantillons contrôlées 10 jours successifs. La courbe globale montre qu'après un déclin brutal survenant entre le premier et deuxième contrôle et témoignant d'une bonne efficacité du piégeage,

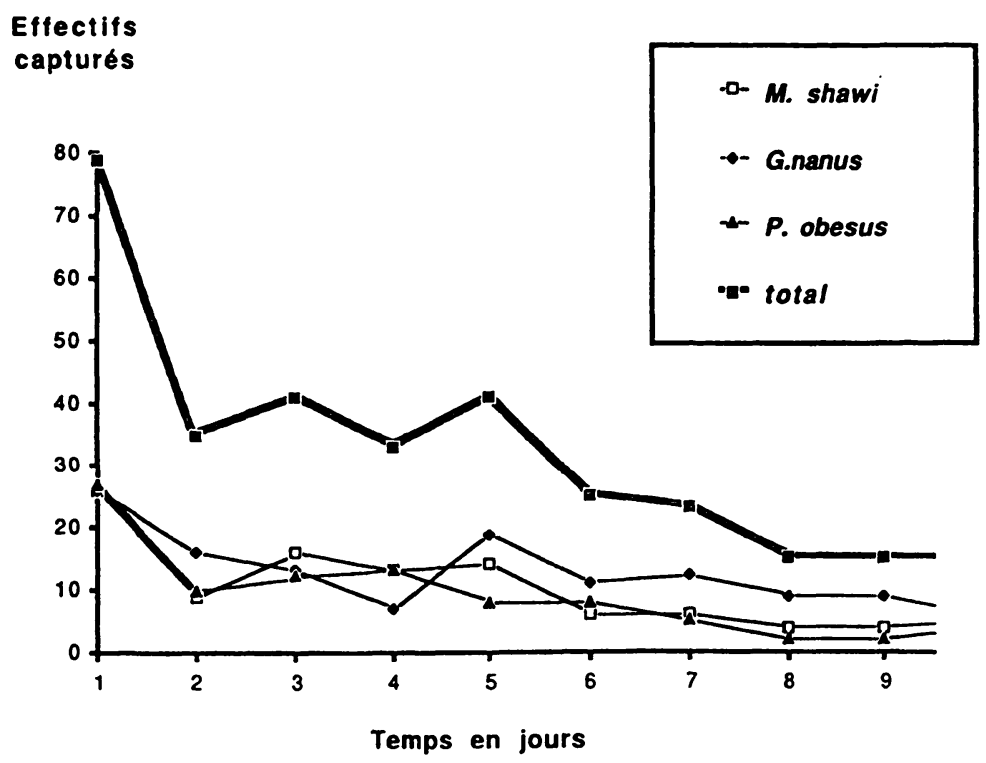

Fig. 5. - Evolution du nombre de captures cumulées sur l'ensemble des bandes-échantillon en fonction du temps.

le nombre de captures se stabilise pendant 4 jours puis décline progressivement sans cependant devenir nul. Ce résultat est le juste reflet de ce que l'on obtient sur chacune des 16 bandes. Les courbes associées à $M$. shawi et $P$. obesus suivent la même tendance. Cet ensemble de données conduirait à conclure qu'afin d'éviter 
une surévaluation de la densité imputable à la recolonisation du milieu vidé artificiellement, phénomène dont l'intensité est généralement densité-indépendante (Lidicker 1985), il conviendrait de limiter la période de piégeage à 5 jours. Cependant, l'examen de la courbe associée à $G$. nanus module cette conclusion. En effet, pour cette espèce, le nombre de captures augmente brutalement le cinquième jour, phénomène que nous avions déjà noté sur le quadrat. Il est probablement à mettre en rapport avec des capacités de déplacement et de colonisation particulièrement développées chez cette espèce. En conséquence, il convient de limiter la durée de la période de piégeage à 4 jours.

TABLEAU 6. - Effectifs capturés sur des bandes posées sur deux milieux et contrôlées pendant 10 jours consécutifs $\left(\mathrm{N}^{\circ}\right.$ : identification de la bande).

Mllien nastoral

\begin{tabular}{|c|c|c|c|c|}
\hline Dotes & $N^{\bullet}$ & M. showi & 6. nanus & P. obesus \\
\hline 19 au & A & 13 & 30 & 7 \\
\hline 2811 & B & 6 & 27 & 9 \\
\hline 18 & E & 7 & 10 & 4 \\
\hline ou & F & 10 & 14 & 2 \\
\hline 27111 & G & 4 & 2 & 3 \\
\hline 11 & E & 3 & O & 1 \\
\hline ou & $\mathbf{F}$ & 3 & 8 & 6 \\
\hline \multirow[t]{2}{*}{$20 \mathrm{IV}$} & G & 4 & 2 & 1 \\
\hline & \multicolumn{4}{|c|}{ Milleu cultivé } \\
\hline 1980 & C & 11 & 2 & 3 \\
\hline 2811 & D & 2 & 12 & 5 \\
\hline 31111 & A & 4 & 8 & 9 \\
\hline ou & B & 3 & 3 & 12 \\
\hline 9 IU & C & 7 & 2 & 4 \\
\hline $22 \mathrm{IU}$ & A & 12 & 12 & 12 \\
\hline ou & B & 14 & 6 & 11 \\
\hline 10 & C & 1 & O & 4 \\
\hline
\end{tabular}

Le tableau 6 récapitule l'ensemble des captures réalisées sur les diverses lignes posées dans les deux milieux dominants, pastoral et agraire. L'hétérogénéité des effectifs capturés ne doit pas surprendre. En effet, elle révèle la diversité des deux milieux principaux.

\section{IV) CONCLUSIONS}

Les deux expérimentations décrites précédemment se sont déroulées au cours d'une phase de forte densité des rongeurs. Cette conjoncture a permis de vérifier que le système de piégeage linéaire proposé n'est pas saturé et peut autoriser, globalement, la capture d'une cinquantaine de rongeurs appartenant aux trois espèces dominantes du peuplement. Ce résultat laisse donc espérer une échelle de mesure de l'indice de densité relativement étendue. Il est vrai qu'elle se réduit au niveau spécifique : 0-17 pour $M$. shawi, 0-10 pour $P$. obesus et 0-24 pour G. nanus (Tab. 5). Pour l'élargir, augmenter le nombre de pièges par carré élémentaire ou augmenter le nombre de contrôles n'aurait guère d'intérêt. En effet, 
ces deux solutions auraient pour conséquence une accélération de la vitesse de capture et induiraient une réduction du temps global de capture afin d'éviter une réinfestation trop massive de la zone piégée. En outre, la seconde solution se heurte à diverses difficultés. Sachant que l'activité de $M$. shawi et de $G$. nanus est essentiellement crépusculaire et nocturne (Zaime 1985), augmenter le nombre de contrôles n'aurait de sens que s'ils sont effectués pendant la nuit, solution qui n'est guère envisageable en raison des conditions de terrain. Instaurer des contrôles supplémentaires diurnes n'intéresserait donc que l'espèce $P$. obesus (Daly et Daly 1973). L'élargissement de l'échelle de mesure passe donc rationnellement par l'augmentation du nombre de carrés élémentaires de la bande-échantillon, soit donc par son allongement. Certaines contraintes du paysage apparaissent alors. En effet, compte tenu de l'effet de lisière supposé, il nous faut déjà disposer de parcelles de dimensions supérieures à $150 \mathrm{~m} \times 60 \mathrm{~m}$. De nombreuses spéculations agricoles locales ne sont d'ores et déjà plus justiciables de cet échantillonnage et il n'est donc guère raisonnable d'opter pour cette solution. En outre, une bande de $100 \mathrm{~m}$ exige la manipulation simultanée de 40 pièges. Augmenter ce nombre rendrait l'opération trop laborieuse. En revanche, la multiplication du nombre de bandes-échantillons nous apparaît comme une solution beaucoup plus intéressante car elle permettrait d'intégrer une éventuelle hétérogénéité du milieu qui aurait échappé à l'observation directe. En outre, cette démarche of fre l'avantage de permettre une exploration qui devrait déboucher sur une meilleure compréhension de l'arrangement spatial des espèces du peuplement de rongeurs et tester l'homogénéité des niveaux de capture.

Les résultats obtenus sur le quadrat ont montré la naïveté de l'hypothèse d'homogénéité du milieu pastoral à l'égard de sa fréquentation par les rongeurs. En particulier, la fréquentation préférentielle de la strate arbustive à $T$. gallica est à mettre en rapport avec son rôle d'abri potentiel, d'une part en période de crue car elle est implantée sur une élévation, d'autre part à l'encontre des prédateurs aviens. Ces " forêts-galeries " s'organisent selon des systèmes linéaires en bordure d'oueds. Par ailleurs, l'orientation des déplacements de rongeurs semble en partie induite par celle des lisières de ces "forêts-galeries ». En conséquence, l'implantation des bandes-échantillons doit tenir compte de cet élément du paysage. De même, dans ce milieu pastoral en évolution rapide, l'implantation de vastes champs de céréales n'est pas sans conséquences sur les déplacements de rongeurs. $M$. shawi et $G$. nanus y trouvent une source alimentaire importante et $P$. obesus des éléments de litière qu'il semble rechercher. Enfin, dans ces écosystèmes où l'eau est si fréquemment un facteur limitant, ne convient-il pas de la prendre en considération lors du choix de l'implantation des bandes-échantillons ? En conséquence, si l'on souhaite réaliser des échantillonnages comparables, il importe de situer les bandes-échantillons à une distance fixe de la rive des oueds et, par là-même, des lisières des "forêts-galeries " de $T$. gallica et de les orienter de façon standard par rapport à la direction de ces structures. On opérera de même à l'égard des champs de céréales. Enfin, il nous faut admettre qu'à l'issue de deux expériences nous ne pouvons prétendre avoir dressé la liste exhaustive des éléments du paysage générateurs d'hétérogénéité dans l'échantillonnage, de même qu'un large champ d'investigations reste ouvert quant à l'éventuelle évolution de l'impact de ces structures au cours du cycle annuel.

La ratière Manufrance semble bien adaptée à $M$. shawi si l'on se fonde sur le taux de recapture et la représentation équilibrée des sexes dans les captures 
et recaptures. Il n'en est pas de même pour $G$. nanus dont les mâles représentent près de $70 \%$ des captures. Ce résultat est-il la conséquence d'un comportement différentiel des deux sexes à un instant particulier du cycle annuel, ou ce phénomène se pérennise-t-il tout au cours de l'année ? Si c'est la première hypothèse qui se révèle correcte, il nous suffira d'en tenir compte dans l'étude diachronique des niveaux de densité. Si c'est la seconde, il nous faudra abandonner l'espoir de pouvoir échantillonner simultanément les 3 espèces dominantes du peuplement au moyen d'un seul type de piège et envisager une modification du piège actuellement utilisé ou l'élaboration d'un piège plus adapté. Un raisonnement analogue peut être développé pour l'espèce $P$. obesus dont les sujets sont peu fréquemment recapturés. Enfin, la mise au point de pièges efficaces, moins onéreux que la ratière Manufrance, deviendra un objectif obligé lors du passage de l'étude intensive locale à l'étude extensive régionale ou nationale.

La durée d'échantillonnage et la fréquence de relevés que nous avons retenus ont été établies à partir de données obtenues sur 5 mois du cycle annuel. Il importera de vérifier le bien-fondé de ce choix à plusieurs époques de l'année. Enfin, l'éventuel impact sur les modalités de capture des relations inter-spécifiques au sein de notre peuplement de rongeurs nous est totalement inconnu.

L'ensemble des résultats exposés ci-dessus a permis de fixer certaines modalités de la méthode d'échantillonnage et de dégager des objectifs de recherche qu'il est indispensable d'atteindre si l'on souhaite la mise au point d'une méthode performante, cheville ouvrière d'études de biologie des populations qu'il est nécessaire de mâtriser pleinement dans la perspective de développer une méthode de lutte intégrée.

\section{REMERCIEMENTS}

Ce travail a reçu un soutien financier de la Fondation Internationale pour la Science.

Nous tenons à remercier pour son accueil la Direction Provinciale de l'Agriculture de Guelmime, pour ses encouragements et soutiens dans bien des domaines, $M$. Ben Halima de la Station antiacridienne d'Ait Melloul, M. F. Rodolphe du Laboratoire de biométrie de l'INRA de Jouy-en-Josas pour nous avoir guidé dans le choix et l'interprétation de certains tests statistiques, et M. F. Spitz pour ses nombreuses suggestions.

\section{BIBLIOGRAPHIE}

DALY, M, , et S. DALY, 1973. - On the feeding ecology of Psammomys obesus (Rodentia, Gerbillidae) in the Wadi Saoura, Algeria. Mammalia, $37: 545-561$.

DALY, M., et S. DALY, 1974. - Spatial distribution of a leaf-eating Saharan gerbil (Psammomys obesus) in relation to its food. Mammalia, 38 : 591-603.

DALY, M., et S. DALY, 1975. - Socio-ecology of saharan gerbils, especially Meriones libycus. Mammalia, 39 : 289-311.

DiJon, R., 1963. - Reconnaissance hydrogéologique et ressources en eau du bassin SayadNoun. Ed. Service géologique du Maroc.

Duplantier, J.M., P. Orsini, M. Thohari, J. Cassaing et H. Croset, 1984. - Echantillonnage des populations de Muridés : influence du protocole de piégeage sur l'estimation des paramètres démographiques. Mammalia, $48: 129-141$. 
KrebS, C.J., et J.H. Myers, 1974. - Population cycles in small mammals. Adv. Ecol. Res., $8: 267-399$.

LIDICKER, W.Z., 1985. - Dispersal. In : Biology of New World Microtus, R.H. TAMARIN ed. Special publication of American Society of Mammalogists, 8 : 420-454.

MAUCHLY, J.W., 1940. - Significance test for sphericity of a normal $n$-variates distribution. Ann. Math. Statist., 11: 204.

PasCal, M., 1984. - Méthode d'échantillonnage d'un rongeur souterrain, le Campagnol terrestre (Arvicola terrestris scherman). Recherche des limites d'utilisation de la méthode. Acta Oecologica, Oecol. Applic., 5 (4) : 303-317.

Pascal, M., et A. Meylan, 1986. - L'échantillonnage linéaire des populations de la forme fouisseuse du Campagnol terrestre (Arvicola terrestris scherman (Shaw)). La Défense des Végétaux, 237 : 3-12.

Petter, F., 1961. - Répartition géographique et écologie des rongeurs désertiques (du Sahara occidental à l'Iran oriental). Mammalia, 25, $\mathrm{N}^{\circ}$ spécial, $222 \mathrm{p}$.

Poulet, A.R., 1982. - Pullulation de rongeurs dans le Sahel. Mécanismes et déterminismes du cycle d'abondance de Taterillus pygargus et Arvicanthis niloticus (Rongeurs, Gerbillidés et Muridés) dans le Sahel du Sénégal de 1975 à 1977. ORSTOM, Paris, 367 p.

Rioux, J.A., F. Petter, D. Akalai, G. Lanotte, A. Ouazzani, M. Séguignes et A. MoHCINE, 1982. - Meriones shawi (Duvernoy, 1842) (Rodentia, Gerbillidae) réservoir de Leishmania major (Yakimoff et Schokhor, 1914) (Kinetoplastida, Trypanosomatidae) dans le sud marocain. C.R. Acad. Sci. Paris, 294 (série 3) : 515-517.

RiouX, J.A., P. RisPail, G. LANOTTE et J. LePART, 1984. - Relation Phlébotomesbioclimats en écologie des leishmanioses. Corollaires épidémiologiques. L'exemple du Maroc. Bull. Soc. Bot. Fr., 131, Actual. Bot. (2/3/4) : 549-557.

RodolPhe, F., et M. PASCAL, 1985. - Méthode d'échantillonnage de la forme fouisseuse du Campagnol terrestre (Arvicola terrestris scherman). Recherche d'un estimateur sans biais de la densité pour un piégeage en bande. Acta Oecologica, Oecol. Applic., 6 (2) : 143-163.

SPITZ, F., 1974. - Démographie du Campagnol des champs, Microtus arvalis, en Vendée. Ann. Zool. Ecol. Anim., 6 (2) : 259-312.

TAITT, M.J., et C.J. KreBS, 1985. - Population dynamics and cycles. In : Biology of New World Microtus, R.H. TAMARIN ed. Special publication of the American Society of Mammalogists, 8 : 567-620.

ZAIME, A., 1985. - Contribution à l'étude écoéthologique de trois rongeurs des milieux aride et semi-aride au Maroc : Meriones shawi, Gerbillus campestris, Lemniscomys barbarus. Thèse Ing. Ecol. Université Rennes I, 230 pp. 